\title{
Advanced Ambulatory Operating Stretcher Learned by Means of Convulational Neural Network (CNN)
}

\author{
${ }^{1}$ Sariena Talpur, ${ }^{2}$ Nazaruddin Khoso \\ ${ }^{1,2}$ Institute of biomedical engineering and technology, Liaquat University of medical and health sciences, \\ Jamshoro Sindh Pakistan \\ sarienatalpur@gmail.com;14bme22@gmail.com
}

\begin{abstract}
According to scientists transportation is the major problem in today's world. The paper presents a method to transferring the patient from long distances or even from shorter distance. Concerned people face many problems in transferring the patient from one place to another place even from ambulance to stretcher or from stretcher to bed and vice versa. Some of the time it is difficult to move the stretcher because of the heavy weight of the patient, It requires more than two or three person in engaging the stretcher while in transferring patient. It will also cause un-peaceful conditions in the hospital and also it will effect on patient's health too.

We tried to overcome these problems via Transfer learning with the help of convolutional neural network in order to move the patients. This type of stretcher solve the problem through self-operating stretcher instead of it operates manually.
\end{abstract}

Keywords: stretcher, ambulatory system, transporting device, ambulance, convolutional neural network.

\section{Introduction}

As the very first we know that stretcher is one of the priority basis need for every hospitals, in order to move patient from ambulance to the stretcher and from stretcher to the respective bed and vice versa [1-4,6-7]. Therefore this is a most essential need for medical aid system. Whenever the emergency occur it causes disturbance in shifting the patients on to the stretcher and stretcher is take it by two or more person in order to reach to its destination place in the hospital $[5,8-10]$. These will all creates massive disturbance in the hospital environment too.

Advanced ambulatory stretcher system is an itself complete device which can gives self-control mobility to the hospitals rather than involvement of any medical staff members. Most of the stretchers can operate on the buttons, hydraulic system or even by the pressure system to control movements and motion of the stretcher $[11,14,16]$. This advanced ambulatory stretcher system is totally based on the artificial intelligence. This system will recognize the objects, person or any distortion that will disturb its path with the help of qualified trained model. Along with this feature it also has small touch screen system that is used to select respective destination in the hospital. By using the transfer learning with 
Sariena Talpur, Nazaruddin Khoso; Advanced Ambulatory Operating Stretcher Learned by Means of Convulational Neural Network (CNN). Journal of Biomedical Engineering and Medical Imaging, Volume 5, No 3, June (2018), pp -34-40

MATLAB software we train the stretcher with convolutional neural network technique along with pretrained ALEXNET model. First it will train then all the movements (right, left, forward, backward, lifting, brake system, horn system) will occur automatically by means of artificial intelligence.

\section{Methodology}

To make the advanced stretcher it consists of four motors for the wheels of the stretcher to provide necessary transportation. The stretcher uses external energy to work therefore we provide the 12 volt battery to the stretcher in order to move it into particular direction. It also uses camera module so that it will recognized the objects.

\subsection{Training of the Stretcher}

ConvNET or CNN (convolutional neural network) is known as one of most famous and successful algorithm for the images and video with deep learning. Similar to the other networks of neural network, $\mathrm{CNN}$ is also composed of three layers input layer, output layer and hundreds and thousands of the hidden layers to acquire the greatest accuracy.

For training the images, CNN uses two of its layers:

- Feature detection layer

- Classification layer

\subsubsection{Feature Detection Layer}

This layer of CNN is consisting of three operations for the purpose to detect the feature of the images or data and perform either one of them.

2.1.1.1 Convulution: It takes input data and applies on them through set of convolutional filters; each filter of convolutional activates respective features from the images.

2.1.1.2 Pooling: Simply identify the output by taking non-linear sampling from input and also reduces other parameters needed for the network to learn about.

2.1.1.3 Rectified Linear Unit(Relu): This the most effective method and also faster than others methods. This is trained by mapping out all -ve values to zero and keep maintaining all +ve values.

These all methods are repeated continuously to hundreds and thousands of the hidden layers to detect features of the input and dataset.

\subsubsection{Classification Layer of CNN}

After passing through feature layer, CNN shift to the next and final layer known as classification layer, whose output is $\mathrm{K}$ dimension vector which represent the number of classes on the training dataset, that network used to predict. 


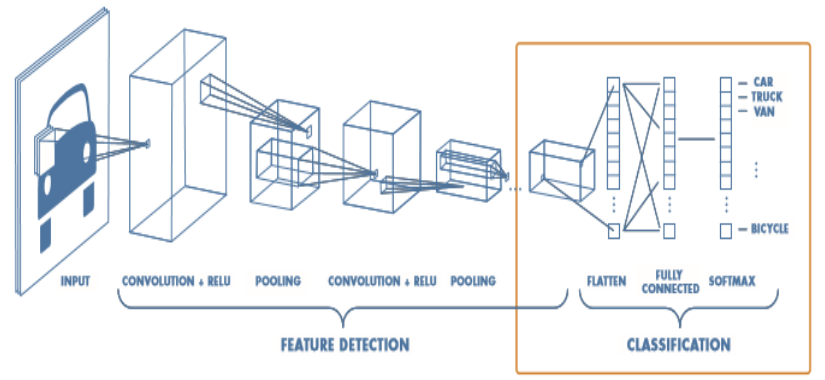

Figure 1: Model of CNN (convolutional neural network)

And we used pre-trained ALEXNET model to makes some changes with the $1^{\text {st }}$ and last three layers for the new dataset of the images. The selection of this model is that it has ability to trained millions of the image at one time and classifies these images into 1000 objects.

Table I: Description of 25 layers of convolution neural network, bold layers indicates working layers.

\begin{tabular}{|c|c|c|c|}
\hline \multicolumn{4}{|c|}{ LAYERS $=25 \times 1$ LAYER ARRAY WITH LAYERS: } \\
\hline 1 & 'data' & Image Input & $\begin{array}{c}227 \times 227 \times 3 \text { images with 'zerocenter' } \\
\text { normalization }\end{array}$ \\
\hline 2 & 'conv1' & Convolution & 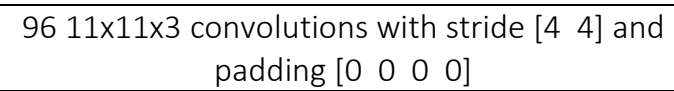 \\
\hline 3 & 'relu1' & ReLU & ReLU \\
\hline 4 & 'norm1' & Cross Channel Normalization & $\begin{array}{c}\text { cross channel normalization with } 5 \text { channels } \\
\text { per element }\end{array}$ \\
\hline 5 & 'pool1' & Max Pooling & $\begin{array}{l}3 \times 3 \text { max pooling with stride }\left[\begin{array}{ll}2 & 2\end{array}\right] \text { and padding } \\
\qquad\left[\begin{array}{llll}0 & 0 & 0 & 0\end{array}\right]\end{array}$ \\
\hline 6 & conv2' & Convolution & $\begin{array}{c}2565 \times 5 \times 48 \text { convolutions with stride [ }\left[\begin{array}{ll}1 & 1\end{array}\right] \text { and } \\
\left.\text { padding [ [ } \begin{array}{lll}2 & 2 & 2\end{array}\right]\end{array}$ \\
\hline 7 & 'relu2' & ReLU & ReLU \\
\hline 8 & 'norm2' & Cross Channel Normalization & $\begin{array}{l}\text { cross channel normalization with } 5 \text { channels } \\
\text { per element }\end{array}$ \\
\hline 9 & 'pool2' & Max Pooling & $\begin{array}{l}3 \times 3 \text { max pooling with stride }\left[\begin{array}{ll}2 & 2\end{array}\right] \text { and padding } \\
\qquad\left[\begin{array}{llll}0 & 0 & 0 & 0\end{array}\right]\end{array}$ \\
\hline 10 & 'conv3' & Convolution & $\begin{array}{c}\left.3843 \times 3 \times 256 \text { convolutions with stride [ } \begin{array}{ll}1 & 1\end{array}\right] \text { and } \\
\text { padding [ }\left[\begin{array}{llll}1 & 1 & 1 & 1\end{array}\right]\end{array}$ \\
\hline 11 & 'relu3' & ReLU & ReLU \\
\hline 12 & 'conv4' & Convolution & $\begin{array}{c}\left.3843 \times 3 \times 192 \text { convolutions with stride [ } \begin{array}{ll}1 & 1\end{array}\right] \text { and } \\
\text { padding [ }\left[\begin{array}{llll}1 & 1 & 1 & 1\end{array}\right]\end{array}$ \\
\hline 13 & 'relu4' & ReLU & ReLU \\
\hline 14 & conv5' & Convolution & $\begin{array}{c}\left.2563 \times 3 \times 192 \text { convolutions with stride [ } \begin{array}{ll}1 & 1\end{array}\right] \text { and } \\
\text { padding [ }\left[\begin{array}{llll}1 & 1 & 1 & 1\end{array}\right]\end{array}$ \\
\hline 15 & 'relu5' & ReLU & ReLU \\
\hline 16 & pool5' & Max Pooling & $\begin{array}{l}3 \times 3 \text { max pooling with stride }\left[\begin{array}{ll}2 & 2\end{array}\right] \text { and padding } \\
\qquad\left[\begin{array}{llll}0 & 0 & 0 & 0\end{array}\right]\end{array}$ \\
\hline 17 & 'fc6' & Fully Connected & 4096 fully connected layer \\
\hline 18 & 'relu6' & ReLU & ReLU \\
\hline 19 & drop6 & Dropout & $50 \%$ dropout \\
\hline 20 & 'fc7' & Fully Connected & 4096 fully connected layer \\
\hline
\end{tabular}


Sariena Talpur, Nazaruddin Khoso; Advanced Ambulatory Operating Stretcher Learned by Means of Convulational Neural Network (CNN). Journal of Biomedical Engineering and Medical Imaging, Volume 5, No 3, June (2018), pp -34-40

\begin{tabular}{|c|c|c|c|}
\hline 21 & 'relu7' & ReLU & ReLU \\
\hline 22 & drop7' & Dropout & Dropout \\
\hline 23 & 'fc8' & Fully Connected & Softmax \\
\hline 24 & 'prob' & Softmax & Classification Output \\
\hline 25 & 'output' & $\begin{array}{c}\text { crossentropyex with 'tench', 'goldfish', and 998 } \\
\text { other classes }\end{array}$ \\
\hline
\end{tabular}

After loading trained model of ALEXNET webcam/camera capture live pictures to identify the distortions like person or objects which can disturb its path.

It used camera /webcam to classify object or data and this all done with MATLAB along with

- Neural network toolbox.

- $\quad$ Support package for using webcam in MATLAB.

- $\quad$ Support package for using ALEXNET.

- LABVEIW along with LEGO MINDSTORM support package.

- Designed stretcher.

\subsection{Programming (VI) for the Stretcher}

To control the stretcher by means of computer it needs software known as LABVEIW 7.1 with LEGO MINDSTORM $^{\circledR}$ NXT software for its programming for giving different direction to the stretcher.it is responsible for the movements.

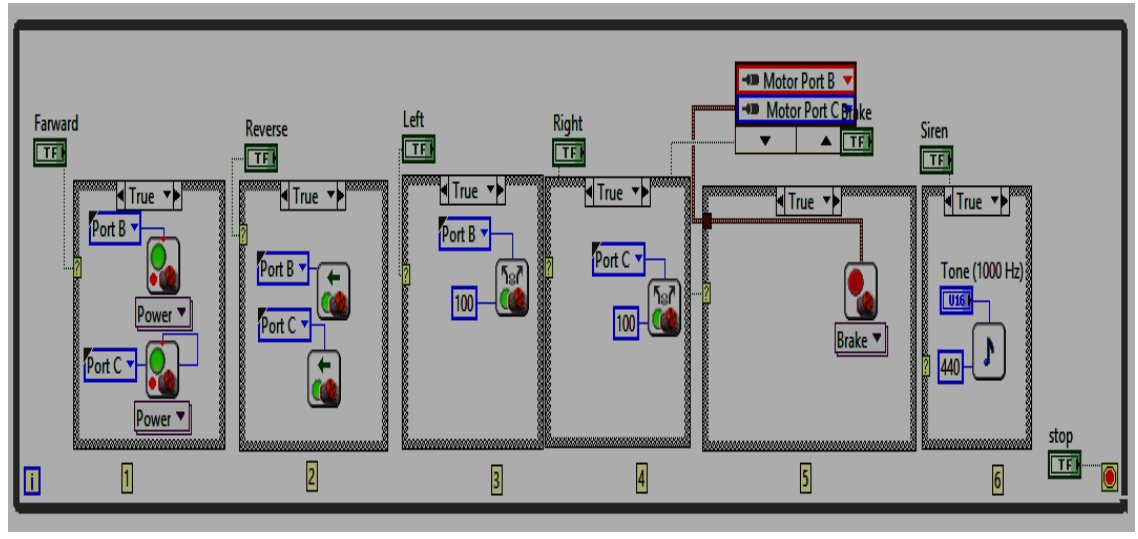

Figure 2: Programming sketch on LabVIEW

This is a complete visualization of programing

Its programming is consist of six sub blocks

o First block is concerned with movement in forward direction. Port B and Port C represent attachment port for respective motors.

o Second block represent movements in backward direction.

$0 \quad$ Third and fourth blocks are responsible for left and right movements.

o Fifth block responsible for braking system whenever obstacle comes in path of stretcher it will stop its movements with help of this block. 
o Sixth and last block is indicating alarming. When obstacle comes in front of the stretcher it will stop and start alarming.

\subsection{Method for Working}

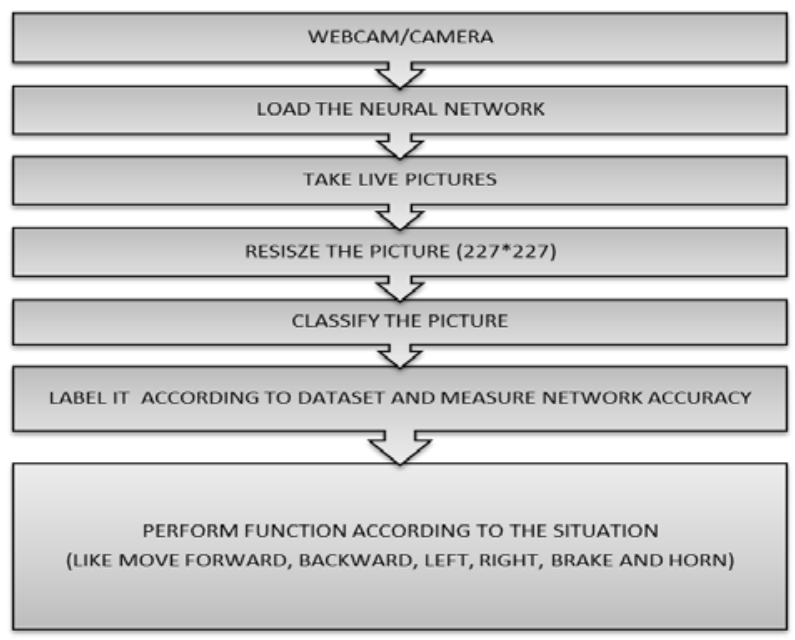

Figure 3: Block diagram showing working principle of CNN based stretcher

\section{Algorithm Architecture}

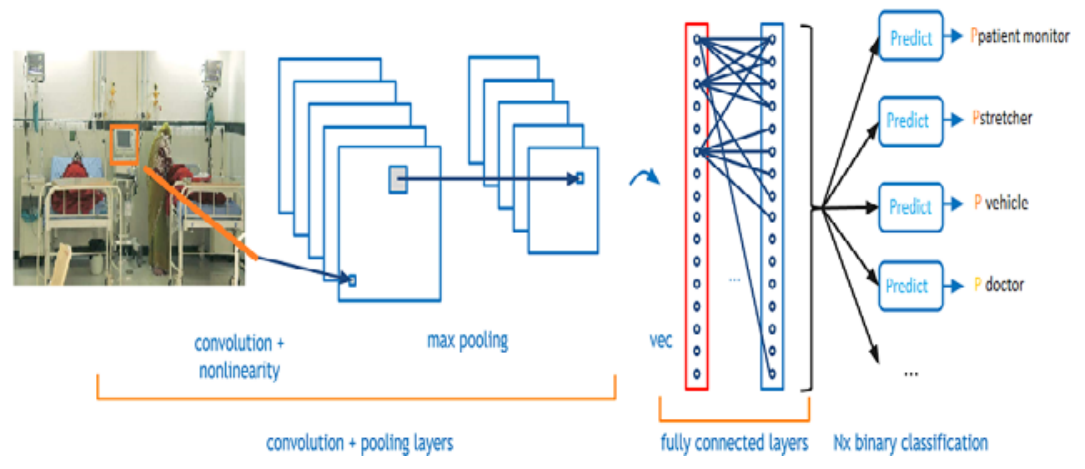

Figure 4: Showing that how CNN based stretcher recognized the objects

Here we have collected thousands of images from hospitals and make a complex dataset of images and to train this dataset and makes trained model for new live images as kernel get predict. The function which we used that is Softmax because it has good accuracy for images dataset instead of others.

$$
Y=\operatorname{softmax}(X \cdot W+b)
$$

Where

$$
\begin{array}{lll}
\mathbf{Y} & =\text { Prediction } & \text { (Output) } \\
\text { Softmax } & =\text { Function } \\
\mathbf{X} & =\text { Input images } & (227 * 227 * 3) \\
\mathbf{W} & =\text { Weight } \\
\mathbf{B} & =\text { Bias }
\end{array}
$$


Sariena Talpur, Nazaruddin Khoso; Advanced Ambulatory Operating Stretcher Learned by Means of Convulational Neural Network (CNN). Journal of Biomedical Engineering and Medical Imaging, Volume 5, No 3, June (2018), pp -34-40

\section{Experiment and Analysis}

Experiments are performed as

- First it will capture live images of the object with the help of camera module.

- Then it recognized the images and decides according to the given condition.

\subsection{Results}

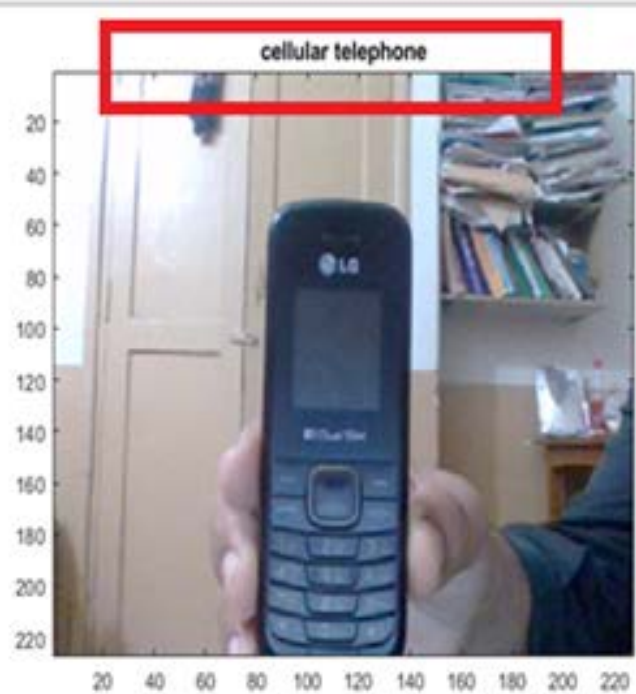

(a)

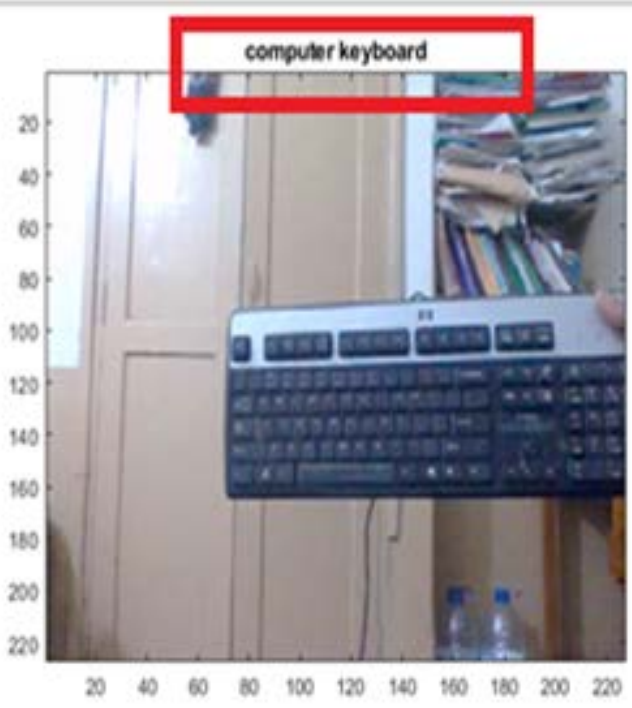

(b)

Figure 5: Indicates the detection of different objects

\section{Conclusion}

Advanced self-operating stretcher system itself has a self-controlling power and self-decision making power. It is suitable for every hospital in the world. This type of stretcher reduces staff stress, maintain environment of the hospitals, reduces the need of staff members to carry stretcher and does not create any massive conditions that will creates any disturbance. This is a complete package needed for every hospital and in the field of health care.

\section{REFERENCES}

[1] Nitin G. Sushir, Ravi B. Bopche, Prof. Hemant M. Bansod, "Design and Fabrication of Wheelchair cum Stretcher" Research Association of Masters of Engineering, Vol:4, pp.6-11, 2017.

[2] Wadgure, A. T., Deshmukh, S., \& Vaidya, R. D. (2014). Development of Modified Mattresses for Patient Handling in Hospital. International Journal of Engineering Trends and Technology, 8(7), 354358. Doi:10.14445/22315381/ijett-v8p264 
[3] Design and Devolvement of modified mattresses for patient handling in hospital. ISSN 2278-0149, VOL 2, NO.4, OCT 2013.

[4] Modeling of modified mattresses for patient handling in hospital. ISSN 2319 - 7293, G.J.E.D.T., Vol. 2(5):41-44. (SeptemberOctober, 2013)

[5] Kevin Hsu ET all fall 2008, a design of Portable Lift for Transferring Wheelchair patients to Elevated Vehicle/ ambulance, University of Michigan Orthotics and Prosthetics Centre.

[6] Ehsanullah Khan et al., Synthesis of Trolley cum Wheelchair for Patient Handling, International Journal of Engineering, Science and Technology (IJEST) Vol.3 No.8, Aug.2011 Pgs.6311-6316.

[7] Wei Ching-Hua et all Nov. 5-8 2007, Hospital bed with Auxiliary Functions of Lateral and Transferring for Immobilized patients Taipei, Taiwan. The 33rd Annual Conference of the IEEE Industrial Electronics Society (IECON) Pgs.2991-2995.

[8] Tiernan John et al., A Survey of the Wheelchair and Seating Market in Irelands, Assistive Technology-shaping the future; AAATE conference proceedings, Vol. 11. No. 1 Pgs- 105-111.

[9] Wadgure, 2(11): November, 2013] ISSN: 2277-9655 Impact Factor: 1.852 http: //www.ijesrt.com(C)International Journal of Engineering Sciences \& Research Technology [32683273]

[10] Wei Ching-Hua et al. (November 5-8, 2007), "Hospital Bed with Auxiliary Functions of Lateral and Transferring for Immobilized Patients Taipei, Taiwan", The 33rd Annual Conference of the IEEE Industrial Electronics Society (IECON), pp. 2991-2995.

[11] Hongbo Wang and Fumio Kasagami, A Patient Transfer Apparatus Between Bed and Stretcher, IEEE transaction on System, Man, and Cybernetics-part-B:, Vol. 38,No.1, Feb.2008.

[12] Rao J. S. 'Mechanism and Machine Theory'- New Age International (p) Ltd Publisher, Edition 2.

[13] Shigley's Mechanical Engineering Design, McGraw-Hill Primis Online, Budynas-Nisbett, Edition 8.

[14] Lesley, Samuel, Porter \& Lucy A, "An Ergonomics Wheelchair for Hemiplegics" Technology \& Disability, Vol.14 No. 4, Pgs 183-189, Publication 2002.

[15] ACEP. New equipment guidelines. Emergency Medical Services. 2001; 30: 42

[16] Alberti G, Cooke M. Ambulance improvement check list.

[17] Allen M. Creating crew space - ambulance modifications for medics. Journal of Emergency Medical Services. 1997; 22: 66-67 